\title{
Implementing evidence-based nursing: some misconceptions
}

During the brief time that we have been engaged in developing Evidence-Based Nursing we have been fascinated by the reactions of friends, professional colleagues, and the media. The overwhelming majority of responses to the concepts of evidence-based nursing and the creation of this journal have been positive. But there have also been misgivings, sometimes generated by misunderstandings. This editorial addresses the following criticisms which we have encountered in person and in print: (1) evidence-based practice isn't new: it's what we have been doing for years, (2) evidence-based nursing leads to "cookbook" nursing and a disregard for individualised patient care and, (3) there is an over-emphasis on randomised controlled trials and systematic reviews in evidencebased health care. We intend the paper to generate, rather than close the debate!

\section{Evidence-based practice isn't new; it's what we have been doing for years}

The plea that "each nurse must care enough about her own practice to want to make sure it is based on the best possible information" is not new. It was written more than 15 years ago. In the same article, Hunt noted that the phrase "nursing should become a research-based profession" had already become a cliché!' Over 20 years ago, Gortner et al lamented the lack of research evidence in many areas of nursing practice, ${ }^{2}$ and the year after, Roper spoke of nursing performing "far too many of its tasks on a traditional base and not within a framework of scientific verifications".

While the recognition of the importance of evidencebased practice is not new, much of the past 20 years has focused on the identification of the barriers to evidencebased practice and the consideration of strategies to overcome these barriers. These barriers include time constraints, limited access to the literature, lack of training in information seeking and critical appraisal skills, a professional ideology that emphasises practical rather than intellectual knowledge, and a work environment that does not encourage information seeking. ${ }^{4}$

We have learned that it is not sufficient to give nursing students a few lectures on the process of doing research and then expect them to use that knowledge throughout their careers in an ongoing process of gathering and interpreting research evidence and implementing findings. Nurses and midwives have been telling researchers for years that they want to deliver research based care, but that they find it difficult to access the research. In a study of health agencies in Ontario, Canada, Mitchell et al found that only 35\% of small hospitals ( $<250$ beds) had nursing research journals in their libraries; $38 \%$ of health agencies based change in nursing practice on the research process; $15 \%$ implemented research utilisation programmes for staff nurses; and 97\% wanted assistance in teaching their nursing staff about research utilisation. $^{5}$
Although we have advocated evidence-based nursing for many years, we have struggled with how to make it happen. This struggle is not unique to nursing but common to all health professions, including medicine. Challenged to address this important issue, an international commitment to evidence-based health care has resulted in a number of initiatives to improve access to research findings such as the Cochrane Collaboration, the Evidence-Based Medicine Working Group, critical appraisal skills teaching programmes, centres for evidence-based practice, research utilisation conferences, and evidence-based journals.

\section{Evidence-based nursing leads to "cookbook" nursing and a disregard for individualised patient care}

In practising evidence-based nursing, a nurse has to decide whether the evidence is relevant for the particular patient. The incorporation of clinical expertise should be balanced with the risks and benefits of alternative treatments for each patient and should take into account the patient's unique clinical circumstances including comorbid conditions and preferences. Those who judge evidence-based nursing as cookbook nursing are ignoring this important component.

The figure shows a simple model for clinical decisions with 4 components that might influence the management of the patient's problems: clinical expertise, patient preference for alternative forms of care, clinical research evidence, and available resources. It is important to note that clinical expertise and patient preference may override the other components of the model for a given decision. For example, clinical expertise must prevail if the nurse decides that the patient is too frail for a specific intervention that is otherwise "best" for his condition, and the patient's preference will dominate when he declines a treatment that clinical circumstances and research evidence indicate is best for his condition. All of these factors, however, have to be considered in the light of the fact that resources for health care are limited. Most decisions in health care have resource implications; there are likely to be occasions when the potential benefit of an intervention is judged to be outweighed by the potential costs.

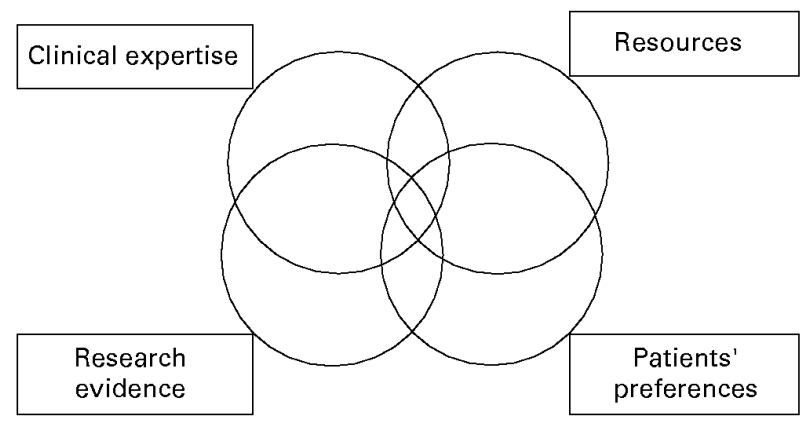

A model for evidence-based clinical decisions (adapted from Haynes et al. ${ }^{6}$ ) 
While individual clinical nurses may not make this judgment, they need to be aware of this important dimension.

In professional training and beyond, we learn the basic mechanisms of disease and pathophysiology and acquire skills in assessment, planning, intervention, and evaluation. We refine these skills by accumulating clinical expertise through observing the correlates and consequences of our actions in dealing with many patients. Many elements of clinical assessment and management require the advanced knowledge that nursing education provides and the expertise that comes with experience.

Evidence from research can help to perfect the expertise but cannot do the examination or sort through the myriad of quantitative and qualitative information that nurses collect during the clinical encounter. Clinical expertise is the crucial element that separates evidence-based nursing from cookbook nursing and the mindless application of rules and guidelines.

Patients have always exercised their preferences for care by choosing alternative treatments, refusing treatment, preparing advance directives ("living wills"), and seeking second opinions. Today's patients have greater access to clinical information than ever before, and some become more knowledgeable about their conditions than their care providers; particularly those with chronic conditions. Although the patient's role in clinical decisions is usually not formalised and is sometimes ignored by care providers, it is an important component of most clinical decisions. Clearly, the best possible scenario is one in which the patient is able to play a full part in making decisions about his or her own health care, having been given an accurate assessment of the current state of knowledge.

Imagine the older person in hospital after a stroke. The nurse caring for this person uses highly developed communication skills, intuition, and clinical experience to get to know the patient and his family. The nurse begins to understand how the patient feels about what is happening to him, and what his goals for rehabilitation might be. As she establishes this rapport, she is in a position to determine if the patient is depressed, anxious, and/or eager to learn about his condition. The relationship that a nurse builds with her patients is important to patients, as shown by Kralik et al in this issue (p63). However, these relationships are enhanced if the nurse is also able to ensure that the caring practices and interventions she uses are safe and effective. Knowing which exercises are effective for patients with hemiplegia, knowing how to prevent their pressure sores, and how to teach them to transfer from a bed to a chair will contribute to high quality patient care.

\section{There is an over-emphasis on randomised controlled trials and systematic reviews in evidence-based health care}

Evidence-based health care is about applying the best available evidence to a specific clinical question. The randomised controlled trial (RCT) is the most appropriate design for evaluating the effectiveness of a nursing intervention, for example the effectiveness of nicotine inhalers in helping patients quit smoking (see Hjalmarson et al in this issue, p45), or the effectiveness of different pressure relieving aids in preventing pressure sores (Vyhlidal et al in this issue, p51). The reason that the RCT is the most appropriate design is that through random assignment of patients to comparison groups, known and unknown confounders are distributed evenly between the groups ensuring that any difference in outcome is due to the intervention.
In a 1997 issue of the British Journal of Nursing, White stated that: "Perhaps the most obviously flawed assumption is that examining research using RCTs is the best way to evaluate the effectiveness of interventions and a better basis for clinical decision-making than the clinical experience of the practitioner." 7

We strongly disagree with White's assertion. History has shown numerous examples of healthcare interventions which, on a patient by patient basis, might appear to be beneficial, but when evaluated using randomised trials have been shown to be of doubtful value, or even harmful. Examples include the use of cover gowns by nurses when caring for normal newborns in the nursery, ${ }^{8}$ and shaving before surgery. ${ }^{9}$ Few of us would want to begin a drug regimen that has not been proved to be safe and effective in a RCT.

More recently, there has been an emphasis on systematic overviews of the research literature. In an overview, eligible research studies are viewed as a population to be systematically sampled and surveyed. Individual study characteristics and results are then abstracted, quantified, coded, and assembled into a database that, if appropriate, is statistically analysed much like other quantitative data. The statistical combination of the results of more than one study, or metaanalysis, effectively increases the sample size and results in a more precise estimate of treatment effect than can be obtained from any of the individual studies used in the meta-analysis. There are 3 overviews in this issue of Evidence-Based Nursing which address the effectiveness of distance medicine technology (Balas et al, p58), compression treatment for venous leg ulcers (Fletcher et al, p50), and psychosocial interventions for children with chronic illnesses (Bauman et al, p43). Through rigorous systematic overviews, nurses are provided with a summary of all the methodologically sound studies related to a specific topic. In most cases, this is much more powerful than the results of any one single study.

Just as randomised trials and systematic overviews are the best designs for evaluating nursing interventions, qualitative studies are the best designs to better understand patients' experiences, attitudes, and beliefs. Results of intervention studies may inform nurses about the optimal effects of an intervention in a sample of patients, but they do not explore and explain the barriers to patient compliance with the intervention, nor how the treatment affects the patient's everyday life, the meaning of illness for the patient, or the adjustment required to accommodate a lifelong treatment regimen. Rigorous qualitative research is based on explicit purposive strategies, in depth analysis of data, and a commitment to examining alternative explanations. In this issue of Evidence-Based Nursing, qualitative studies examine parents' experiences with a critically ill child in the paediatric intensive care unit ( $\mathrm{Mu}$ and Tomlinson, p60); patients' experiences of nursing (Kralik et al, p63); experiences of long term oxygen therapy (Ring and Danielson, p64); women's role as carers (Wuest, p62); and patterns of violence experienced by homeless battered women (Clarke, p61).

Through Evidence-Based Nursing we hope to convey that good evidence does involve more than RCTs and systematic overviews. Each research design has its purpose, its strengths, and its limitations. The key is ensuring that the right research design is used to answer the question posed.

The ultimate goal of nursing is to deliver to patients the best available care. Despite numerous barriers to using research there continues to be strong motivation among nurses to learn the skills required to practice evidence-based 
nursing. The application of research findings to practice goes hand in hand with clinical expertise and with patient preferences and values. The types of study designs that form the basis for evidence-based practice will vary depending on the nature of the question asked.

We have attempted to identify and address common misconceptions about evidence-based nursing. Readers are encouraged to comment and to let us know if there are additional impediments to this relatively new initiative in nursing.

School of Nursing,

ALBA DiCENSO, RN, PhD

Faculty of Health Sciences,

McMaster University,

Hamilton, Ontario, Canada

Centre for Evidence Based Nursing,

NICKY CULLUM, RN, PhD

Department of Health Studies,

University of York, UK
School of Nursing,

Faculty of Health Sciences,

McMaster University,

Hamilton, Ontario, Canada

1 Hunt J. Indicators for nursing practice: the use of research findings. J Adv Nurs 1981;6:189-94

2 Gortner SR, Bloch D, Phillips TP. Contributions of nursing research to patient care. J Adv Nurs 1976;1:507-18.

3 Roper N. Justification and use of research in nursing. I Adv Nurs 1977;2:365-71.

4 Royle JA, Blythe J, Ingram C, et al. The research utilisation process: the use of guided imagery to reduce anxiety. Canadian Oncology Nursing Journal 1996;6:20-5.

5 Mitchell A, Janzen K, Pask E et al. Assessment of nursing research utilization needs in Ontario health agencies. Can J Nurs Admin 1995;8:77-91.

6 Haynes RB, Sackett DL, Gray JAM, et al. Transferring evidence from Haynes RB, Sackett DL, Gray JAM, et al. Transferring evidence from
research into practice. 1. The role of clinical care research evidence in clinical decisions [editorial]. ACP Journal Club 1996 Nov-Dec;125:A14-6.

7 White S. Evidence-based practice and nursing: the new panacea? British Journal of Nursing 1997;6:175-7.

8 Rush J, Fiorino-Chiovitti R, Kaufman K, et al. A randomised controlled trial of a nursery ritual: wearing cover gowns to care for healthy newborns. Birth 1990;17:25-30.

9 Hoe NY, Nambiar R. Is preoperative shaving really necessary? Ann Acad Med Singapore 1985;14:700-4.

\section{LETTERS}

Setting the results of individual studies in context

Felicitations on the birth of Evidence-Based Nursing $(\mathrm{EBN})$ and best wishes for the future. I was particularly glad to see that you have made a start on working out how to select reliable studies reporting qualitative data.

The first issue of EBN prompts me to write with a suggestion which I hope you will consider implementing as the journal matures. I think it is potentially misleading to concentrate on abstracting individual studies (as EBN has begun to) unless either the authors or the commentators have made a systematic effort to set the results of these studies within the context of other relevant studies. For example, the study on the effects of sucrose on crying in newborn babies which you selected for abstracting is 1 of at least 5 relevant controlled trials, yet these other trials are not mentioned in the commentary. In fact, the relevant trials on this topic have been assessed in a good systematic review which must have been published at about the same time as the single study you selected for presentation. ${ }^{1}$ In my view, the systematic review provides evidence of much greater importance to your readers than the outcome of the particular study you selected (in which the phrase 'Ross Special Formula' is mentioned three times!).

Unless the results of the individual studies you select for presentation in EBN are systematically set in proper context, either by the authors or by your commentators, your readers are likely to be misled-by the play of chance among similar studies if by nothing else.

IAIN CHALMERS, MSc (Soc Med) Director, UK Cochrane Centre, Oxford, UK.

Editors' response:

Thank you for welcoming the first issue of $\mathrm{EBN}$ and for taking the time to give us this thought provoking feedback. Your letter raises important issues that we must grapple with as the journal develops. We agree that it is potentially dangerous to present the findings of isolated studies when there is an existing systematic review.

We endeavour to bring relevant, existing systematic reviews to the attention of each commentator, who is briefed to set the new research in the context of existing knowledge. Unfortunately, in this case the metaanalysis by Stevens et al became available after we had gone to press and the commentator was unable to refer to it in her commentary. Nevertheless, your letter is a useful reminder of the importance of setting individual study results in context. EvidenceBased Nursing places high priority on abstracting recent systematic reviews of relevance to nursing, and we hope to increase our coverage as more nursing related systematic reviews become available.

1 Stevens B, Taddio A, Ohlsson A, et al. The effi-
cacy of sucrose for relieving procedural pain
in neonates-a systematic review and meta-
analysis. Acta Paediatr 1997;86:837-42.

Evidence-based mental health

Firstly, I would like to welcome the publication of Evidence-Based Nursing in that it provides, through the abstracts and commentaries, a useful method of finding a way through the mass of information currently deemed essential reading if nurses are to apply research findings to practice.

I am particularly pleased to see the qualitative studies which attempt to explore, as Anne Mulhall highlighted in the EBN notebook, the meaning of situations for researcher and subject, meanings which, as she says, cannot easily fit into the notion of scientific, quantitative research methods, essential as these may be in other areas.

I was disappointed to see, however, that although some of the studies did focus on what might be called psychosocial aspects of health and illness, few of them focused primarily on mental health and illness. Does this reflect a dearth of research studies in this field? Unless such matters are addressed more fully, I feel that the journal will have little appeal to mental health nurses in the UK and will miss an opportunity to promote research-based practice in an area where it has perhaps, traditionally, been lacking.

\section{MAXINE KEMPSTER RN, BSc}

Poole, Dorset, UK

Editors' response:

Thank you for taking the time to give us feedback. We are endeavouring to ensure that although this is a general journal, it contains something of interest for everyone. We are certainly keen to find ways of ensuring that we identify good quality research concerned with mental health and illness and in this issue you will find papers on grief intensity after pregnancy loss, and the impact of prenatal alcohol exposure on behaviour and learning problems in adolescence. It would be helpful if you could review the list of journals that we have read to identify studies for inclusion in Evidence-Based Nursing. If there are any journals that report studies related to nursing and mental health that we are currently not reviewing, we would be pleased to know about them. You might also like to know that a new international, multidisciplinary journal, EvidenceBased Mental Health was launched by the BMJ Publishing Group and the Royal College of Psychiatrists in February 1998. All studies considered for that journal are also reviewed for possible inclusion in Evidence-Based Nursing. 